\title{
A coarse grained model for DNA and polymer packaging: statics and dynamics
}

\author{
I. ALI $¥ \|$, D. MARENDUZZO $* *$, C. MICHELETTIף\# \# and J.M. YEOMANS $\$ * *$ \\ $\nmid$ Department of Physics, College of Science, PO Box 36, Al-Khod 123, Sultan Qaboos University, Oman \\ \$Mathematics Institute, University of Warwick, Coventry CV4 7AL, UK \\ ๆ International School for Advanced Studies (S.I.S.S.A.) and INFM, Via Beirut 2-4, 34014 Trieste, Italy \\ $\S$ The Rudolf Peierls Centre for Theoretical Physics, 1 Keble Road, Oxford OX1 3NP, UK
}

\begin{abstract}
We present a numerical characterization of the statics and dynamics of the packaging of a semi-flexible polymer inside a sphere. The study is motivated by recent experiments on the packaging of DNA inside viral capsids. It is found that the force required to confine the coarse-grained polymer is in fair agreement with that found in experiments for the packaging of the phi29 bacteriophage genome. Despite its schematic nature, the model is capable of reproducing the most salient dynamical features of packaging experiments such as the presence of pauses during individual packaging processes and the trend of the resisting force as a function of chain packed fraction.
\end{abstract}

Keywords: DNA packaging; Statistical mechanics; Polymer physics; Bacteriophages

Msc: 82D60: Statistical Mechanics; Polymers; 92C05: Biophysics

\section{Introduction}

A crucial issue of the mechanism through which viruses infect bacteria regards the means of delivery of the viral genome inside the host. It has been recently suggested [1] that the delivery (or ejection) does not involve any "active" cellular machinery but relies on the high pressure with which the DNA is packed inside the viral capsid [1-5].

Recent single molecule experiments on the phage phi29 have clarified several aspects of the DNA packaging in capsids [3]. In particular, the study of Smith et al. [3] revealed that the portal motor which packages the DNA into phi29 in vivo has to oppose a large internal force (exerted by the already-loaded genome) which can be as high as $50 \mathrm{pN}$. The same study also showed that the incremental force required to load an additional portion of DNA in the capsid does not increase monotonically with the fraction of already-packed genome. Besides these equilibrium aspects of the genome loading process the experiments have provided a dynamical characterization of the packaging process establishing that (a) the packaging rate is not constant as a function of the loaded genome and that (b) "pauses" in the packaging are present in any individual experiment. No mechanism was proposed to explain the presence of these pauses.

In this study we discuss a simplified coarse-grained model of the DNA packaging process which reproduces the experimental findings summarized above. The qualitative agreement with experimental data shows, $a$ posteriori, that considerable insight into complicated biochemical processes, such as bacterial infection, can be gained through the use of schematic models amenable to numerical simulations.

\section{Model and methods}

Within the simplified packaging model considered here, the viral capsid is described as a spherical hull with a hole at the top which accomodates the motor (see figure 1). The viral genome is modelled as a semi-flexible polymer, made of $N$ equispaced beads (monomers). The spacing of the beads centres is equal to the diameter of the beads, $\sigma$. The latter is taken to match the experimentally-measured hydration radius of double-stranded DNA, $\sigma=2.5 \mathrm{~nm}$. The instantaneous spatial arrangement of the polymer at time $t$, is therefore entirely specified by the coordinates of

*Corresponding author. Tel.: +44-24-76574831. Fax: +44-24-76524182. Email: davide@maths.warwick.ac.uk

||Tel.: +968-24415456. Fax: +968-24414228. Email: issam@squ.edu.om.

\#Tel.: + 39-0403787300. Fax: +39-0403787528. Email: michelet@ sissa.it.

**Tel.: + 44-1865273992. Fax: +44-1865273947. Email: j.yeomans1@physics.oxford.ac.uk.

Journal of Theoretical Medicine

ISSN 1027-3662 print/ISSN 1607-8578 online (C) 2005 Taylor \& Francis Group Ltd

http://www.tandf.co.uk/journals

DOI: $10.1080 / 10273660500149927$ 


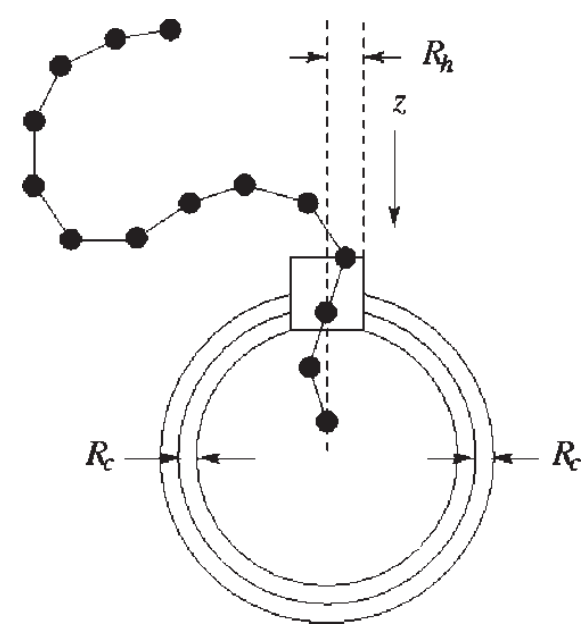

Figure 1. Packaging of a chain into a capsid: $R_{s}$ is the capsid radius, $R_{h}$ is the radius of the hole and $R_{c}$ is the critical distance from the capsid surface, below which a repulsive Lennard-Jones force is applied. The motor, and the range over which it applies its force, is denoted by the cylinder of radius $R_{h}$ at the capsid entrance.

the beads centres, $\left\{\vec{r}_{i}(t)\right\}, \quad i=1, \ldots, N$. Non-consecutive pairs of beads interact through a pairwise potential, $V(r)=\epsilon /(r / \sigma)^{12}$. Consistently with previous studies we set the interaction coefficient equal to $\epsilon=k_{B} T$ [2]. Besides this term, which is meant to reproduce the screened repulsion of DNA phosphate charges, we introduce a bending rigidity:

$$
V_{\mathrm{b}}=-\kappa \sum_{i=2}^{N-1}\left(\vec{r}_{i+1}-\vec{r}_{i}\right) \cdot\left(\vec{r}_{i}-\vec{r}_{i-1}\right)
$$

Due to the large computational expenditure involved in the repeated detailed characterization of the packaging kinetics, it is not feasible to consider polymers constituted by more than 100 beads. Accordingly, we have set $N=$ 100. Besides the contour length, thus corresponding to $L=250 \mathrm{~nm}$, another relevant length scale is set by the persistence length $\xi=\sigma \kappa / k_{B} T$. In this study, in order to have the contour length much larger than the persistence length we set $\kappa=5 \quad k_{B} T$, corresponding to a persistence length of $\sim 12.5 \mathrm{~nm}$. This choice, due to practical numerical considerations, underestimates the dsDNA persistence length $(\sim 50 \mathrm{~nm})$. For this reason, the comparison of our findings with the experiments will mostly be qualitative.

The packaging action of the motor protein is reproduced by applying a constant downward force to any monomer unit in the neighborhood of the motor region (see figure 1). In our simulations we have set $R=7.25 \mathrm{~nm}$ for the capsid radius and $f_{0}=16.6 \mathrm{pN}$ for the motor force (close to the minimum needed for the packaging). We note that our parameters lead to a filling fraction, measured as the ratio between the total volume of the polymer beads and the capsid volume, of $\sim 0.5$.

The dynamical evolution of the system was followed through the stochastic rotation algorithm [6], which allows to model the hydrodynamic interactions of the polymer and surrounding solvent. The details of the algorithm are discussed in [6,7]. The strength of hydrodynamic interactions can be quantified by the dimensionless parameter $h$ [7], which is $\sim 0.3$ with our parameters.

\section{Results}

We present our results in terms of the loading curves, $f(x)$, which represent the force, $f$, opposing the motor action, as a function of the fraction of the chain already packaged, $x$.

The result of a typical run is shown in figure 2. It can be seen that the curve rises quite sharply after $x=0.5$. Also, the rate of increase of the force is smaller near $x=1$. The shape of the force curve is in qualitative agreement with experiments [2]. Experiments also show a milder increase of the force opposing the motor action near full packing. The maximum of the curve is at $\sim 50 \mathrm{pN}$ in experiments and slightly less than $30 \mathrm{pN}$ in the simulations (1 simulation unit here is $\sim 1.67 \mathrm{pN}$, we measure the force acting on the first bead within the hole, along the positive $z$ axis, which is opposite to the motor force direction). Comparing the free energy lost during packing and that gained by ejecting the chain (which is obtained by switching off the motor force) indicates that about $30 \%$ of the motor work during packaging is dissipated by dynamic effects.

Figure 3(a) shows a typical plot of the packed bead fraction as a function of time in a single run. We observe pauses in which the number of packed beads is constant. These are washed out by averaging. Figure 3(b) shows how the packing rate decreases with $x$. The qualitative shape of figure $3(b)$ can be guessed by assuming a quasistatic over-damped dynamics for $x, \mathrm{~d} x / \mathrm{d} t=\Gamma\left(f_{0}-f(x)\right)$, with $\Gamma$ a damping constant (compare figures 2 and $3(b)$ ).

Correlating the pause times with the instantaneous configuration of the polymer is useful to understand their nature. By inspection of the packaging process two types of pauses can be recognised. First, there are brief pauses

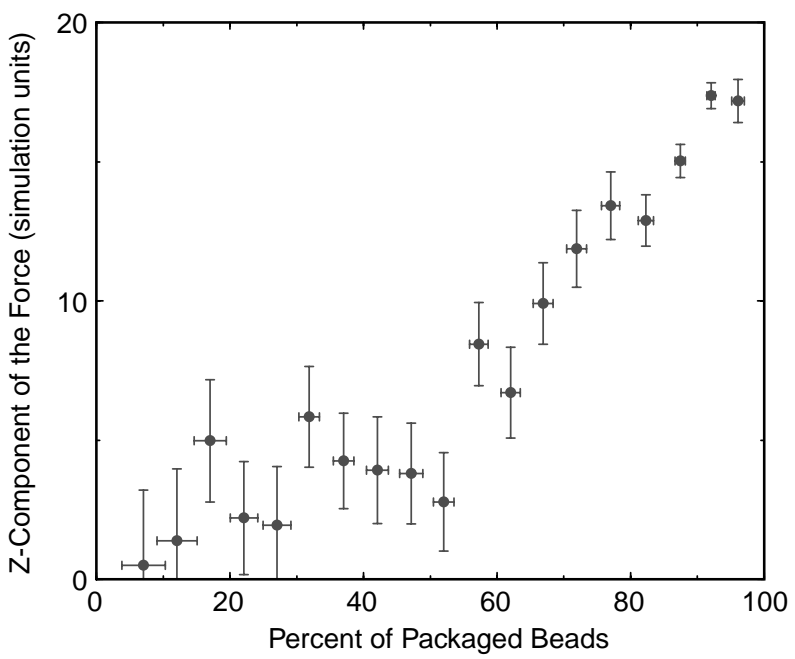

Figure 2. Component of the force opposing the motor force direction, exerted by the semi-flexible chain bead closest to the motor, as a function of packaged polymer. 

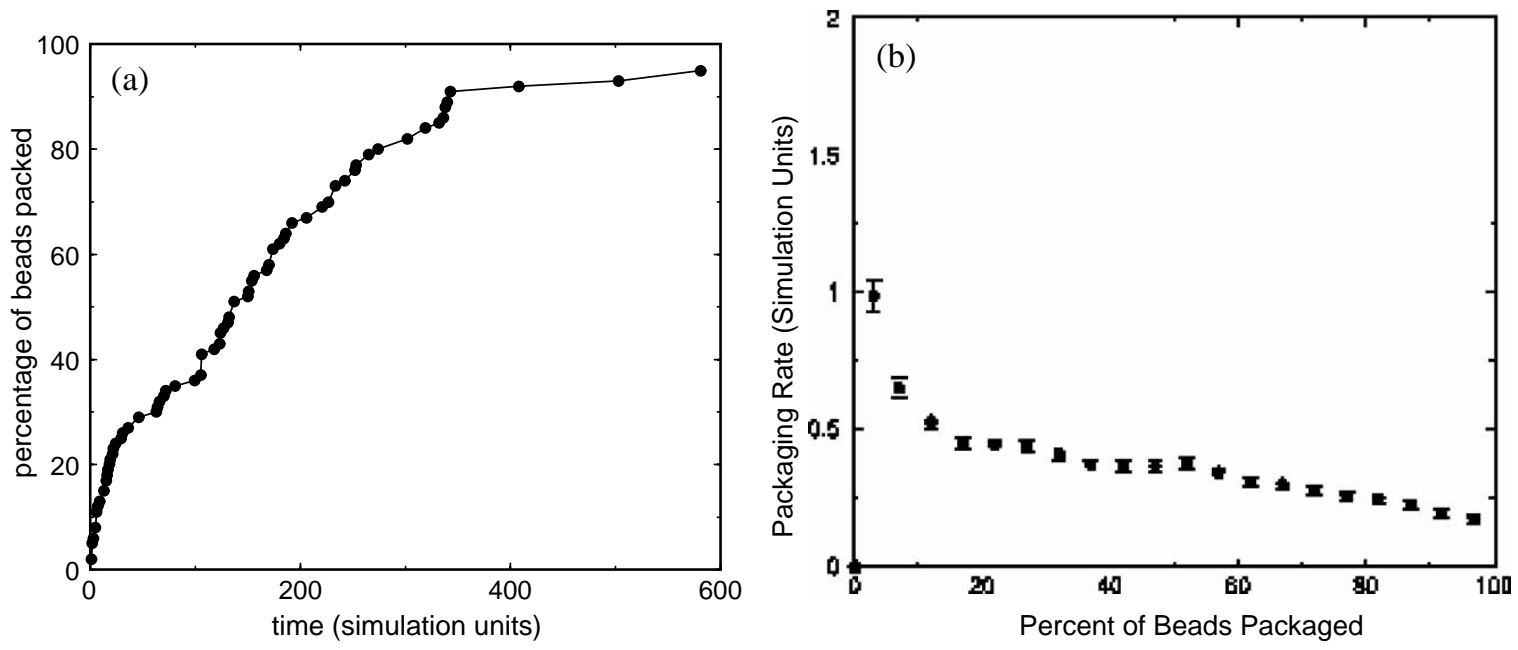

Figure 3. (a) Number of packaged beads versus time, in simulation units, for a single packaging run for a semi-flexible polymer. (b) Packaging rate versus time (both in simulation units) for a semi-flexible polymer (averaged over 62 runs).

when there is only a small fraction of packaged chain. These are due to the fact that the tail of the polymer outside the capsid is not optimally placed close to the hole for the monomers to be fed in and thus must reorient before packaging can proceed. Second, there are longer and more frequent pauses near full packing. These are due to the fact that the polymer's instantaneous configuration exerts a large pressure on the entering beads due to local crowding. Indeed we find that pauses correlate with the relaxation of internal pressure. Pauses are also found in fully flexible chains. Our simulations suggest that these pauses are not of special biological origin but are rather connected to the difficulty of rearranging a chain in a crowded environment.

A movie of the packaging process (for a flexible chain) can be viewed online.

\section{Conclusions and discussion}

In conclusion we have presented a coarse grained model for the packaging of a polymer. Despite its simplicity, we get semi-quantitative agreement with experimental data on (a) the magnitude of the force exerted by the DNA during the packaging; and (b) the packaging rate as a function of packed genome. We also observe pauses in single runs as in [1].

It thus seems that the present approach-amenable to numerical treatment in present day computers-can capture the salient physics of DNA packaging inside bacteriophage capsids. It would be interesting to pursue this approach further and to characterize the dynamics of DNA ejection from a phage, and eventually to describe in a coarse-grained way the key stages of phage mediated bacterial infection.

\section{Acknowledgements}

We thank M. A. Webster for helpful discussions.

\section{References}

[1] Purohit, P.K., Kondev, J. and Phillips, R., 2003, Mechanics of DNA packaging in viruses. Proc. Natl Acad. Sci. USA, 100, 3173-3178.

[2] Kindt, J., Tzlil, S., Ben-Shaul, A. and Gelbart, W.M., 2001, DNA packaging and ejection forces in bacteriophages. Proc. Natl Acad. Sci. USA, 98, 13671-13674.

[3] Smith, D.E., Tans, S.J., Smith, S.B., Grimes, S., Anderson, D.L. and Bustamante, C., 2001, The bacteriophage phi29 portal motor can package DNA against a large internal force. Nature, 413, $748-752$.

[4] Arsuaga, J., Tan, R.K.Z., Vazquez, M., Sumners, D.W. and Harvey, S.C., 2002, Investigation of viral DNA packaging using molecular mechanics model. Biophys. Chem., 101, 475-484.

[5] Marenduzzo, D. and Micheletti, C., 2003, Thermodynamics of DNA packaging inside a viral capsid: The role of DNA intrinsic thickness. J. Mol. Biol., 330, 485-492.

[6] Malevanets, A. and Kapral, R., 1999, Mesoscopic model for solvent dynamics. J. Chem. Phys., 110, 8605-8613.

[7] Ali, I., Marenduzzo, D. and Yeomans, J.M., 2004, Dynamics of polymer packaging. J. Chem. Phys., 121, 8635-8641. 


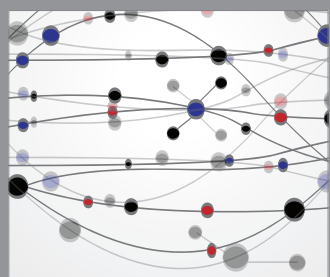

The Scientific World Journal
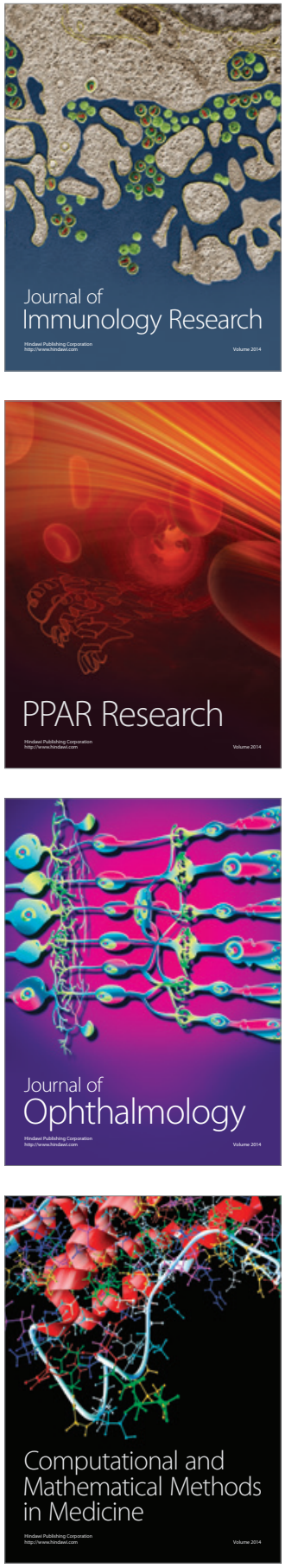

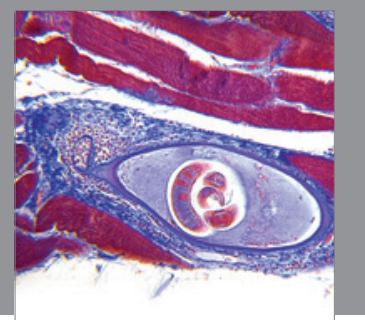

Gastroenterology

Research and Practice
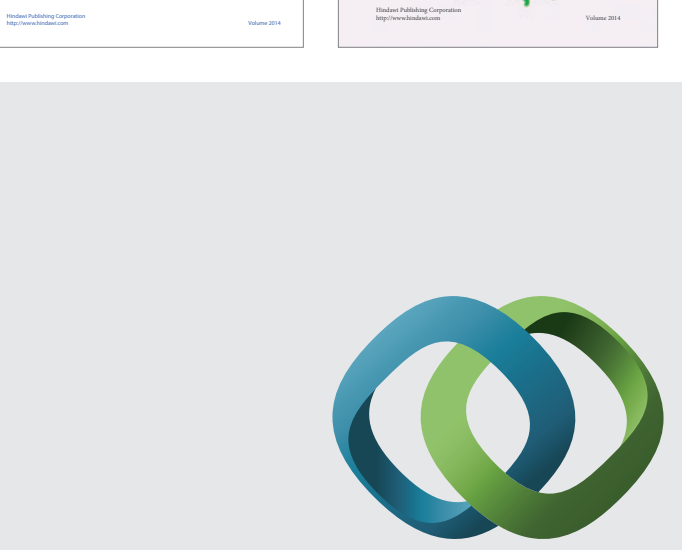

\section{Hindawi}

Submit your manuscripts at

http://www.hindawi.com
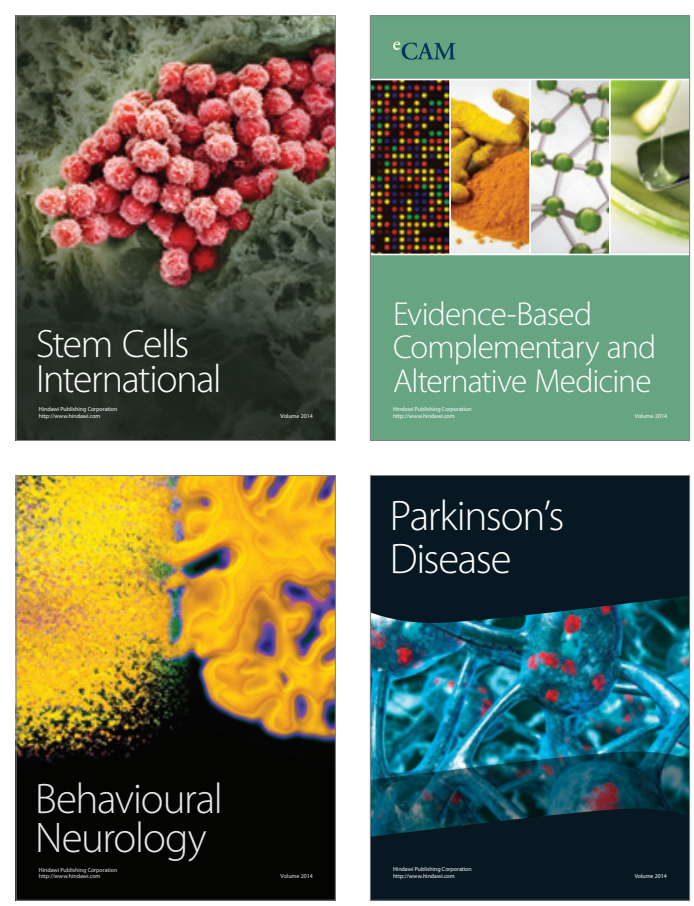

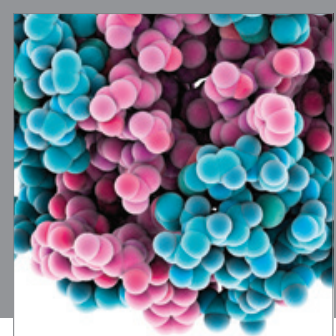

Journal of
Diabetes Research

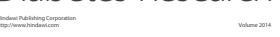

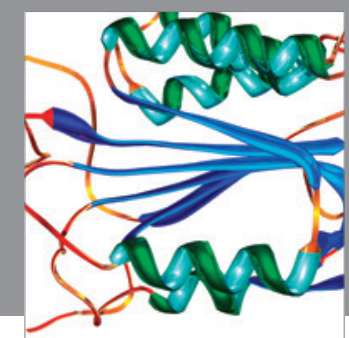

Disease Markers
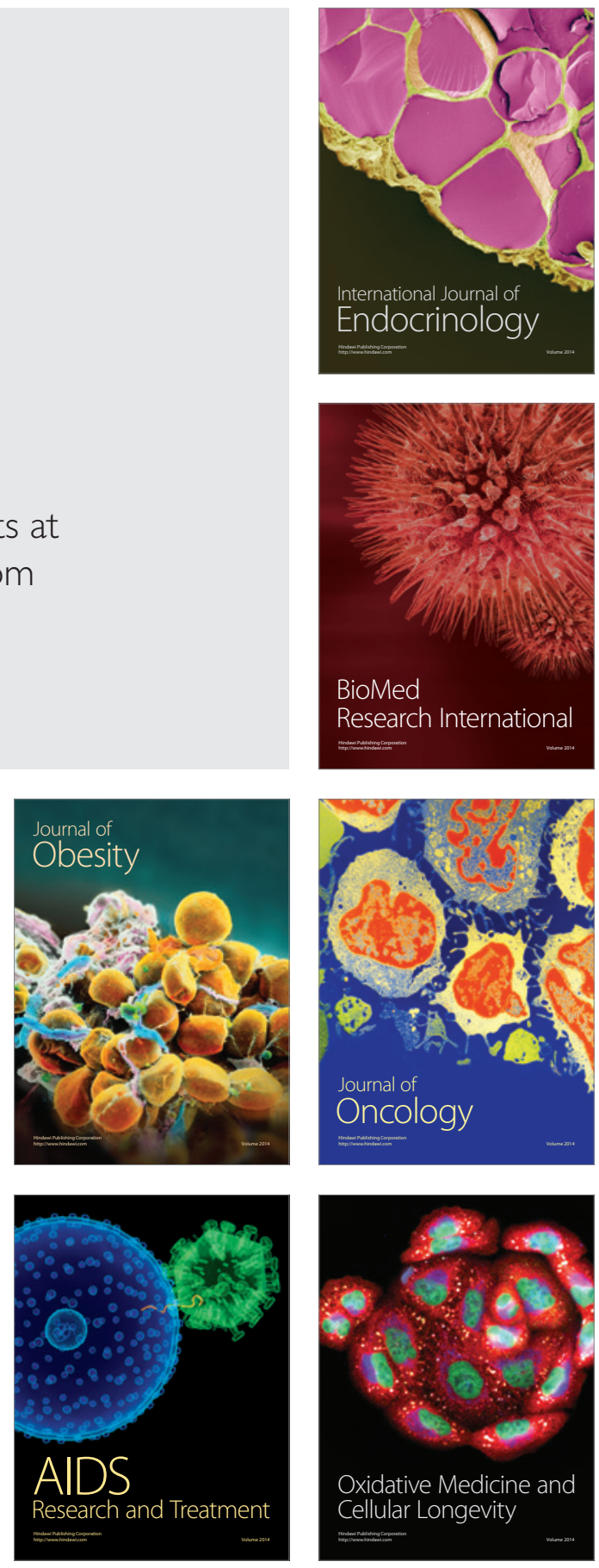